\title{
The venue management problem: setting staffing levels, shifts and shift schedules at concession stands
}

\author{
Oyku Ahipasaoglu ${ }^{1} \cdot$ Nesim Erkip ${ }^{2} \cdot$ Oya Ekin Karasan ${ }^{2}$
}

Published online: 26 July 2018

๑) Springer Science+Business Media, LLC, part of Springer Nature 2018

\begin{abstract}
The aim of this research is to determine staffing requirements as well as shift scheduling, with the objective of maximizing the expected contribution from the venue. We specifically consider theaters where demand at concession stands is non-stationary, being largely dominated by the showtimes, the days of the week and the movie types. Determination of the number of workers and their shift in such facilities can be considered as a venue management problem. As one of the distinctive characteristics of our study, we maximize the expected contribution of venue operations while combining shift scheduling in a mathematical model. In addition to the objective function, shift scheduling differs from that in the standard literature, as shift beginning and end times are not restricted. Because of the highly variable demand in the venue management problem considered, flexible shifts are used, with flexible beginning and end times, as long as workers meet the specifications of the type to which they belong. We prove that the venue management problem under consideration is NP-complete. Under mild conditions, we show that the binary requirements of some of the variables in the model can be relaxed. Computational results of a case study are provided.
\end{abstract}

Keywords Venue management $\cdot$ Revenue $\cdot$ Tour scheduling $\cdot$ Shift scheduling

\section{Introduction}

In theaters, demand at concession stands is non-stationary, being largely dominated by showtimes. Demand peaks can be observed in particular before shows and during intermissions, with low to almost no demand at other times. In parts of Europe, movies in large (multiplex) theaters are shown with a 10-min intermission, during which demand peaks are frequently observable; thus management of concession stands becomes a critical issue. Additionally, since there is a limited amount of time before movies and during the breaks, it

Nesim Erkip

nesim@bilkent.edu.tr

Oyku Ahipasaoglu

oyku.ahipasaoglu@gmail.com

Oya Ekin Karasan

karasan@bilkent.edu.tr

1 The Boston Consulting Group, 34394 Levent, Istanbul, Turkey

2 Department of Industrial Engineering, Bilkent University, 06800 Bilkent, Ankara, Turkey is important to serve and satisfy the customers during these 10-15-min periods in order to receive revenue.

Demand at concession stands is also highly variable within a weekly period. More demand can be observed during evenings than daytime and during weekends than on weekdays. Since movie schedules are usually updated on Fridays, it is possible to consider the demand at concession stands as cyclic — with a weekly cycle from Friday to Friday in this case.

The operation of revenue-making service facilities in an airport resembles that of concession stands. Again, the demand for the service facility is a function of other schedules, and is highly variable within a day and between days of the week. Determining the number of workers and their shifts can be considered a venue management problem as well.

The aim of this research is to turn the above-described venue management problem into a tour scheduling problem and to determine staffing requirements and worker shifts, with the objective of maximizing the net contribution from the concession stands.

Worker scheduling has a long history in the operations research literature. Ernst et al. (2004a, b), Alfares (2004) and Van den Bergh et al. (2013) all provide detailed reviews 
and bibliographies of worker scheduling literature and group them according to problem classifications, application areas and solution methods. Demand modeling, days-off scheduling, shift scheduling, tour scheduling, task assignment, shift assignment and workforce planning are all problem classifications under worker scheduling. Worker scheduling covers a wide range of applications including transportation (airline, bus and railway), call centers, nurse scheduling, healthcare services, financial services and manufacturing. To tackle these problems, a vast array of exact and heuristic solutions are employed.

In one of the earlier attempts, Buffa et al. (1976) in their seminal work proposed the following systematic approach to worker scheduling, and most subsequent research literature followed this approach:

1. Forecast demand,

2. Convert demand forecasts into staffing requirements

3. Schedule shifts

4. Roster workers to shifts.

In this study, we focus on steps 2 and 3 simultaneously. As one of the distinctive characteristics of our study, we optimize staff requirements (rather than ensuring that we reach a certain service level). Hence, we maximize the contribution of venue operations (contribution defined as revenue minus cost of workers) while combining shift scheduling in a mathematical model. This is one of the major differences in a venue management problem compared to a more standard formulation, where labor costs are minimized subject to a given service requirement. In addition to the objective function, shift scheduling is differs from the standard literature in that beginning and end times of shifts are not restricted, allowing us to start a shift for a worker at any time (possibly limited to some reasonable values). Given the high variability in demand in the venue management problem considered, flexible shifts are used, with flexible beginning and end times, as long as the amount of time worked meets the requirements for the specific worker types.

Section 2 summarizes the relevant literature and details the problem we consider. In Sect. 3, we present and discuss a mathematical model for the stated problem. The formulation contains constraints satisfying the requirements for different types of workers in terms of the number of days and specific labor regulations and contractual agreements. We prove two results: the first is an optimality cut on the formulation, and the second is a structural property that relaxes some of the binary variables in the model as continuous under the concavity assumption of the revenue function. In Sect. 4, we present an approach for modeling demand and venue operations considered as the case under study. In Sect. 5, we present the computational complexity status of the problem and show that even in its simplest form, it results in an NP-complete problem. In Sect. 6, we provide the results of extensive computational studies to show the efficacy of the approach for the case study and to provide insights. Lastly, Sect. 7 summarizes the contributions of the research and presents conclusions.

\section{Details of the venue management problem considered and literature review}

We consider a venue management problem where expected revenue received per unit of time is a function of the number of workers present at that time. We solve the contribution (expected revenue less staff costs) maximization problem while setting shifts to be utilized, schedules of shifts and staffing levels for each shift. Workers are available with varying capacities and costs, called types. Examples are full-time (with different hour requirements/week, overtime possibilities and costs), part-time (with different hour requirements/week and costs) and other possibilities which apply to venues considered. We assume that workers are homogeneous with respect to skill. In other words, they can serve an equal number of customers per unit time, but they vary with respect to the number of hours worked as well as their work patterns.

We assume that there are several worker types, where a type is identified by the working days (not necessarily consecutive) in the week and the number of working hours per workday shift. A particular worker type works the same number of hours per working day. However, two shifts of the same type have the flexibility to start at different times on two different working days, i.e., may have differing patterns. For example, there might be full-time workers taking weekends off and working $8 \mathrm{~h}$ per day. These workers may start the shift at 8:00 a.m. on Monday, take noon off and finish off at 5:00 p.m. Some of these workers may follow Monday's pattern on Tuesday, and some may start their shift at 9:00 a.m. on Tuesday and finish off at 6:00 p.m. Each worker type will also have an associated wage for the planning horizon.

Time-related constraints are imposed to ensure that the regulations and the total workload designated for each type are satisfied.

As the problem is contribution maximization, we are not expected to be concerned with over- or understaffing. However, if needed, constraints on various service levels can be included in the model.

The demand for venue management considered is stochastic and dynamic. Thus, a transient analysis of such queues is needed to determine the demand for the concession stands during different breaks. This adds further challenges to our problem.

General personnel scheduling problems are covered extensively in the literature. Numerous problem types are considered, and often many commonalities exist between different 
classifications. Van den Bergh et al. (2013) has a recent review paper that covers various types of problems discussed in the literature. We study the literature for the above problem under various subtitles: tour scheduling; shift scheduling; scheduling problems for venues as well as for hospitality and tourism, and retail; and related problems with regard to profit maximization objectives. We complete the literature review by summarizing the closely related work and issues regarding systems with stochastic and dynamic demand.

In tour scheduling problems, off-days and shift schedules are determined for a roster horizon. Brusco and Jacobs (2000) and Rekik et al. (2004) provide compact formulations for the tour scheduling problem where tours are considered to be continuous. Ours is a type of tour scheduling problem where tours are not continuous, however, as the venue considered does not operate $7 / 24$. We consider possible working patterns where daily start times may vary for a worker, and hence consider a weekly plan, resulting in a "tour." Jacobs and Brusco (1996) formulate a weekly tour scheduling problem with a compactly described constraint set. Brusco et al. (1995) present a tour scheduling problem where there are restrictions for allowable work patterns. Cezik et al. (2001) consider constraints due to a union contract and describe the associated constraints in a network flow framework. In contrast to previous studies, they are able to handle the restriction on the difference between the start times of two consecutive shifts. In all of the above studies, unlike our our work, the staff demand requirements to be satisfied are fixed, and the objective function is to minimize the total labor cost. While covering demand, Felici and Gentile (2004) consider a staff satisfaction maximization objective in which the likability of two consecutive pairs of shifts is expressed by a positive weight. The authors also study the associated polyhedron. With our flexible shift characterization, we can handle all of the above-mentioned limitations on the work patterns.

The shift scheduling problem, a subproblem of the tour scheduling problem, is another topic of interest, where shifts are planned with details such as breaks. Typically, shift structures are pre-specified. Thompson (1995) is an early and a comprehensive example. Our flexible shifts identified by type and start time are of a similar flavor to those in this study. Aykin (2000) compares different modeling approaches for the problem. van Veldhoven et al. (2016) consider a personnel shift scheduling problem with more complicated shift structures. Mattia et al. (2014) propose a robust optimization framework for shift scheduling, motivated by call centers where robustness is considered over workforce demand values. Extending the above problem, Dahmen and Rekik (2014) consider heterogeneous workers, whereas Boyer et al. (2013) consider a shift scheduling problem with complicated task structures and heterogeneous workers. Defraeye and Van
Nieuwenhuyse (2016a) propose a branch-and-bound algorithm for shift scheduling under stochastic non-stationary demand. Again, in all of these studies, workforce demand requirements to be satisfied are fixed, and thus the objective function is to minimize costs.

With regard to the area of application, as described by Ernst et al. (2004b), the problem we consider fits to a venue management problem largely arising in airport-related staff scheduling. However, hospitality, tourism or retail sectors are reasonable fits as well. The literature on venue management and hospitality and tourism problems contains a number of examples for determining staffing levels for different applications. These include determining staffing levels at a police communication center Chen (2000), immigration personnel scheduling for given flight schedules Littler and Whitaker (1997), personnel scheduling for a fast food establishment Love and Hoey (1990), airport personnel planning Mason et al. (1998), freight handling personnel at an airport Nobert and Roy (1998), personnel scheduling truck loading/unloading with a known schedule Sarin and Aggarwal (2001), scheduling of airport personnel by an airline Schindler and Semmel (1993), and scheduling of cleaning crews for airlines Stern and Hersh (1980). However, none of the above studies represents a system with a direct revenuemaking situation.

We now review studies where the motive is to maximize profit, and hence the workload to be covered becomes a decision variable rather than a constraint. One stream of the literature does not deal with shift scheduling problem, but determines staffing requirements under various assumptions regarding demand and other pertinent issues. Ren and Zhou (2008) consider the role of effort exerted relative to increased profits. Lam et al. (1998) use a sales response model with store traffic forecasting to determine the size of the workforce. Similar ideas are implemented by Mani et al. (2015) for retail environments and Hampshire et al. (2009) for call centers. Chapados et al. (2014) study a retail store environment from the perspective of sales staff scheduling to maximize profits. The expected revenue curves are linearized after a detailed demand/revenue modeling. The attempt is to model expected revenue as a function of the number of workers; we take a similar path. They make use of automata and constraint programming in order to represent feasible shifts as words over an alphabet. As a contribution, we allow the use of such general revenue function within a direct and more efficient mathematical programming framework.

Another stream of literature attempts to obtain a solution to the tour scheduling problem, like we do. One of the early attempts to deal with this problem is Thompson (1997). The study considers an incremental structure for each additional worker affecting the service beyond a minimum level, instead of using a revenue function, which does 
not fit to the applications considered in that study. Kabak et al. (2008) propose a two-stage model: In the first stage, a sales response model is used as an input to a mathematical programming model. In the second stage, a mathematical program with new input is solved iteratively through the use of a simulation model for the validation of the sales response function. The mathematical programming model is a cost minimization model, with the flexibility of hourly wages. An arbitrary number of fixed shifts can be considered, and both part-time and full-time workers are allowed. Note that hourly requirements come from the sales response model. Finally, Helber and Henken (2008) consider a profitoriented shift scheduling problem for a call center. Here, the demand process is quite sophisticated, allowing for stochastic dynamic demand with skills-based routing, impatient customers and retrials. However, the shift scheduling problem considered is relatively simple, allowing for only a small number of shift types. One can see that there are some similarities between the above studies and our work. Nevertheless, the problem we described at the beginning of this section has features which are not covered by any work mentioned above.

Various structures of demand are considered in the literature. In general, we consider a stochastic dynamic demand structure with abandonment. Studies by Littler and Whitaker (1997) and Sarin and Aggarwal (2001) have similarities to ours with respect to demand structure, as they deal with a very variable demand flow that is a function of other decisions (not necessarily independent or exogenous). Additionally, in our problem, stochastic variability of demand is relatively high. For example, we observe abandonment, which is not the case in airport operations. Aksin et al. (2007) review possible abandonment behaviors, and consider a linear relation between waiting time and the probability of abandonment. The issue of finding customer demand requires a thorough analysis of the associated stochastic process for demand. Please refer to a recent review by Defraeye and Van Nieuwenhuyse (2016b), where approaches for determining non-stationary demand structures for service are considered for staffing and scheduling decisions. Our problem differs from the above literature, as we are merely seeking transient results.

In our models, we identify worker types by the number of workdays per week and working hours per workday. This approach is flexible enough to incorporate different types of shift pattern requirements that arise from considering human factors as well as work regulations. Unlike the bulk of the existing literature, we schedule workforce so as to generate revenue. With the realistic assumption that the revenue generated will be a concave function of the workforce, we are able to show that the binary requirement of a large number of variables can be relaxed in the search for the optimal solution, resulting in significant CPU time savings.

\section{Notation and mathematical model}

In this section, we provide some background information on our problem and introduce the notation that will be adopted throughout the text.

Let $\mathcal{S}=\{1, \ldots, S\}$ represent a set of time-cells. In our case study, the planning horizon is 1 week. Within the week, the workers work a specific number of days, and within a day, a specific number of hours. In other words, the time-cells correspond to hour-day combinations, i.e., $S=14 \times 7$ assuming that there are 14 working hours per day. However, depending on the planning horizon and the modularity of the shifts, the definition of time-cells can change. $\mathcal{D}=\{1, \ldots, D\}$ is the set of days in the planning horizon, $\mathcal{Q}=\{1, \ldots, Q\}$ is the set of available workers at any time-cell, and $\mathcal{T}=\{1, \ldots, T\}$ is the set of different types of workers available. Note that, for the sake of convenience, we keep track of the time-cells and days separately. Also note that we allow for an arbitrary number of working hours for a worker type. We assume that each day he/she has the flexibility of starting at any time of day (given that the considered type is working that day), provided that there are a sufficient number of hours to work (specified by the worker type on that day) until the closing time. For example, a worker type might work only on Tuesdays and Wednesdays, each with $4 \mathrm{~h}$. Any worker with this work pattern is allowed to start anytime on those days within the first 11 time-cells. Another worker type might work on Tuesdays and Thursdays for $4 \mathrm{~h}$ each. With this approach, we do not have to explicitly consider breaks, as they can be embedded in the type information.

Parameters:

$$
\begin{aligned}
D^{t}= & \text { set of days a type } t \text { worker works during the planning } \\
& \text { horizon, } t \in \mathcal{T}, \\
I^{t}(d)= & \text { set of available starting time-cells for a type } t \text { worker } \\
& t \in \mathcal{T} \text { during day } d \in D^{t}, \\
I^{t}(s)= & \text { set of starting time-cells which make type } t \text { worker, } \\
& t \in \mathcal{T}, \text { available during time-cell } s, s \in \mathcal{S}, \\
C^{t}= & \text { salary of each type } t \text { worker for the planning horizon, } \\
& t \in \mathcal{T}, \\
\bar{W}_{s}= & \text { threshold value for the waiting time, } s \in \mathcal{S}, \\
\bar{L}_{S}= & \text { threshold value for the number of lost customers, } \\
& s \in \mathcal{S}, \\
\bar{N}_{s}= & \text { threshold value for the number of customers waiting } \\
& \text { longer than } \bar{W}_{s}, s \in \mathcal{S} .
\end{aligned}
$$

For every $s \in \mathcal{S}, k \in \mathcal{Q}$ :

$W_{s k}=$ expected waiting time per customer if $k$ workers are used in time-cell $s$, 
$L_{s k}=$ expected number of customers lost if $k$ workers are used in time-cell $s$,

$N_{s k}=$ expected number of customers to wait more than $\bar{W}_{s}$ if $k$ workers are used in time-cell $s$,

$V_{s k}=$ expected revenue generated if $k$ workers are used in time-cell $s$.

An employee type $t$ is identified by the specific days, $D^{t}$, and the specific time-cells in those days, $\cup_{d \in D^{t}} I^{t}(d)$, that workers of this type may start work.

Decision variables:

$x_{s k}= \begin{cases}1, & \begin{array}{l}\text { if the number of workers working in time-cell } s \\ \text { is } k\end{array} \\ 0 & \text { otherwise }\end{cases}$ $s \in \mathcal{S}, \quad k \in \mathcal{Q}$,

$y_{s}^{t}=$ number of type $t$ workers starting work on time-cell $s$ $t \in \mathcal{T}, \quad s \in \mathcal{S}$,

$\lambda^{t}=$ number of type $t$ workers to be paid during a planning horizon $t \in \mathcal{T}$.

We shall assume that revenue is a non-decreasing function of the number of workers, and that waiting times and number of lost customers are non-increasing functions of the number of workers. In other words, we have:

$V_{s k} \leq V_{s k^{\prime}}, \quad W_{s k} \geq W_{s k^{\prime}}, \quad L_{s k} \geq L_{s k^{\prime}} \quad$ and

$N_{s k} \geq N_{s k^{\prime}} \quad s \in \mathcal{S}$, and $k, k^{\prime} \in \mathcal{Q}: k \leq k^{\prime}$.

The venue management problem seeks a profit-maximizing workforce schedule that satisfies the service level requirements. To this end, the following integer programming (IP) model determines, for each worker type $t$ and for each working day $d \in D^{t}$ of this worker type, the size of the workforce and their daily start times.

$$
\begin{array}{ll}
\max & \sum_{s \in \mathcal{S}, k \in \mathcal{Q}} V_{s k} x_{s k}-\sum_{t \in \mathcal{T}} C^{t} \lambda^{t} \\
\text { s.t. } & \sum_{k \in \mathcal{Q}} x_{s k} \leq 1 \quad s \in \mathcal{S}, \\
& \sum_{k \in \mathcal{Q}} k x_{s k} \leq \sum_{c \in I^{t}(s), t \in \mathcal{T}} y_{c}^{t} \quad s \in \mathcal{S}, \\
& \sum_{s \in I^{t}(d)} y_{s}^{t} \leq \lambda^{t} \quad d \in D^{t}, \quad t \in \mathcal{T}, \\
& \sum_{k \in \mathcal{Q}} W_{s k} x_{s k} \leq \bar{W}_{s} \quad s \in \mathcal{S}, \\
& \sum_{k \in \mathcal{Q}} L_{s k} x_{s k} \leq \bar{L}_{s} \quad s \in \mathcal{S},
\end{array}
$$

$$
\begin{aligned}
& \sum_{k \in \mathcal{Q}} N_{s k} x_{s k} \leq \bar{N}_{s} \quad s \in \mathcal{S}, \\
& x_{s k} \in\{0,1\} \quad s \in \mathcal{S}, k \in \mathcal{Q}, \\
& y_{s}^{t} \geq 0, \quad \text { integer } \quad s \in \mathcal{S}, \\
& \lambda^{t} \geq 0, \quad \text { integer } \quad t \in \mathcal{T} .
\end{aligned}
$$

The objective function (1) corresponds to the profit for the planning horizon, which is the total revenue generated less the wages to be paid to the workers. Constraints (2) and domain restrictions (8) jointly ensure that the value of variable $x$ will index the number of workers working for each time-cell of the planning horizon. Note that we allow for some time-cells to have no workers if it benefits the overall profit. Constraints (3) ensure that the number of workers counted as working during time-cell $s$ cannot exceed the number of workers whose daily working hours coincide with the specific cell $s$. Specifically, the left-hand side of these constraints corresponds to the size of the workforce on time-cell $s$, and the right-hand side sums the number of workers that work at time $s$, i.e., and their start times ensure that they will work through time $s$. Constraints (4) guarantee that the number of workers of each type working in the planning horizon, i.e., $\lambda^{t}$ value, cannot be lower than the number of workers starting each day. Constraints (5)-(7) jointly enable the intended quality of service to the customers. Finally, (8)-(10) are domain restrictions.

Without loss of generality, we may assume that the same number of workers of a given type will start work every working day particular to that type. More formally,

Lemma $1 \sum_{s \in I^{t}(d)} y_{s}^{t}=\lambda^{t}$ is an optimality cut to (1)-(10) for each $d \in D^{t}, t \in \mathcal{T}$.

Proof Note that an optimality cut for a model is a constraint which is satisfied by at least one optimal solution of this model. Even though there would be feasible solutions violating this constraint, we may add it to the constraint set of the model without changing the optimal value.

Let $(x, y, \lambda)$ be an optimal solution to (1)-(10). Assume for some $\hat{t}$ and $\hat{d} \in D^{\hat{t}}$, inequality (4) is not tight, i.e.,

$\sum_{s \in I^{\hat{t}}(\hat{d})} y_{s}^{\hat{t}}<\lambda^{\hat{t}}$

Since both $y$ and $\lambda$ are integral vectors, the difference $\lambda^{\hat{t}}-$ $\sum_{s \in I^{\hat{t}}(\hat{d})} y_{s}{ }^{\hat{t}}$ is integer-valued. Let $\hat{s} \in I^{\hat{t}}(\hat{d})$. Then, $(x, \bar{y}, \lambda)$, where

$\bar{y}_{s}^{t}= \begin{cases}y_{s}^{t} & \text { for } \quad s \neq \hat{s}, t \neq \hat{t} \\ y_{s}^{t}+1 & \text { for } \quad s=\hat{s}, t=\hat{t}\end{cases}$

solves (1)-(10) optimally as well. Proceeding in this fashion, alternative optimal solutions can be attained satisfying the constraint set (4) tightly. 
Lemma 1 states that inequalities (4) can be replaced with the following equations:

$\sum_{s \in I^{t}(d)} y_{s}^{t}=\lambda^{t} \quad d \in D^{t}, t \in \mathcal{T}$

In our computations, we replaced inequalities (4) with equalities (11).

Lemma 2 Constraints (5)-(7) in the above model can be replaced with an equivalent form

$\sum_{k \in \mathcal{Q}} k x_{s k} \geq \bar{\lambda}_{s} \quad s \in \mathcal{S}$

where $\bar{\lambda}_{s}=\min \left\{k \in \mathcal{Q}: W_{s k} \geq \bar{W}_{s}, L_{s k} \geq \bar{L}_{s}\right.$ and $N_{s k} \geq$ $\left.\bar{N}_{s}\right\}$.

Proof Each of the constraints (5)-(7) provides a minimum service level requirement on the number of workers. Constraint (12) simply replaces these three constraints with the bottleneck service level requirement.

In addition to the optimality cut provided by Lemma 1 , the integer programming model (1)-(10) has a strong structural property when the revenue is a concave function of the number of workers. In particular, once the decision variables $y$ 's take integer values, the binary nature of the index $x$ variables can be relaxed. We shall prove this property for a general IP form, which will then imply the result for the IP in consideration.

Consider the following integer programming model, say M,

$$
\begin{array}{ll}
\max & \sum_{i=1}^{n} f(i) u_{i} \\
\text { s.t. } & \sum_{i=1}^{n} u_{i} \leq 1 \\
& \sum_{i=1}^{n} i u_{i} \leq b_{1} \\
& \sum_{i=1}^{n} i u_{i} \geq b_{2} \\
& u \in\{0,1\}^{n} .
\end{array}
$$

where $f:\{1, \ldots, n\} \rightarrow \mathbb{R}_{\geq 0}$ and $b_{1}, b_{2} \in\{1, \ldots, n\}$ with $b_{2} \leq b_{1}$. Let RM be the linear programming relaxation of this model where constraints (17) are replaced with $0 \leq u_{i} \leq$ $1 i \in\{1, \ldots, n\}$.

Theorem 1 If $f$ is a concave function on $\{1, \ldots, n\}$ and $b_{1}, b_{2} \in\{1, \ldots, n\}$ with $b_{2} \leq b_{1}$, then there exists an optimal solution to $R M$, say $u^{*}$, such that $u^{*} \in\{0,1\}^{n}$.
Proof Let $\tilde{u} \notin\{0,1\}^{n}$ be an optimal solution to RM. We shall construct $u^{*} \in\{0,1\}^{n}$ as an alternative optimal solution.

Define $u^{*}$ as

$u_{i}^{*}=\left\{\begin{array}{ll}1 & \text { for } i=b_{1} \\ 0 & \text { for } i \neq b_{1}\end{array}\right.$.

Clearly, $u^{*}$ is feasible for M. Moreover, since $f$ is a concave function,

$\sum_{i=1}^{n} f(i) u_{i}^{*}=f\left(b_{1}\right) \geq f\left(\sum_{i=1}^{n} i \tilde{u}_{i}\right) \geq \sum_{i=1}^{n} f(i) \tilde{u}_{i}$

and hence $u^{*}$ is also optimal for M.

For every time-cell $s \in \mathcal{S}$, define the following functions:

$\mathcal{V}_{s}: Q \rightarrow \mathbb{R}_{\geq 0}$ such that $\mathcal{V}_{s}(k)=V_{s k}$ for every $k \in Q$

Corollary 1 If for every $s \in \mathcal{S}, \mathcal{V}_{s}$ is a concave function on $\mathcal{Q}$, then the integrality of binary $x$ variables in (1)-(10) can be relaxed.

Proof Note that once the $y$ and $\lambda$ variables are fixed, the model (1)-(10) decomposes for each $s \in \mathcal{S}$. For a feasible $y, \lambda$ to (1)-(10) and for a fixed $s \in \mathcal{S}$, let $n=Q, f(i)=$ $V_{s i}, u_{i}=x_{s i}$ for every $i \in\{1, \ldots, n\}, b_{1}=\sum_{c \in I^{t}(s), t \in T} y_{c}^{t}$ and $b_{2}=\bar{\lambda}_{s}$. Then, for this fixed $s$, the model (1)-(10) projected onto the $x$ variables becomes a model of the form $M$ for which Theorem 1 implies the desired result. Note that we should implicitly assume $\bar{\lambda}_{s} \leq b_{1}$, for otherwise there is no feasible solution.

Note that concavity of $f$ is indeed necessary for Theorem 1 to hold. Consider the small example where $f(1)=$ $1, f(2)=2, f(3)=4, b_{1}=2$ and $b_{2}=0$. Then, $\left(0,0, \frac{2}{3}\right)$ solves RM optimally with an objective value of $\frac{8}{3}$ which is strictly greater than the optimal value of $\mathrm{M}$, which is 2 .

\section{Modeling demand and venue operation}

Venues that are considered in this work do not exhibit a consistent demand pattern in the classical sense. Specifically, daily demand for the concession stand in a movie theater exhibits a huge variance, making it possible to label it even as erratic. However, when one analyzes the pieces that make up the total demand for a concession stand, a simpler picture can be observed: What drives the demand for a concession stand during the intermission of a certain movie is a function of (i) the type of the movie, (ii) time of the day, (iii) day of the week and (iv) week of the year. Venue managers will most 
likely have sufficient data to estimate the probability distribution of the number of incoming customers to the venue and the proportion of those asking for service at a concession stand during intermission as a function of the type of movie and its showtime. Moreover, it has been observed that the revenue obtained per customer served can be assumed to be dependent only on the type of movie, practically constant per customer. As a result, demand for customers visiting a concession stand during an intermission is time-dependent and stochastic. Given this distinction, we model total demand by analyzing demand for each intermission of each movie and build up for the concession demand for each time-cell. For us, it is important that we can compute the demand/serviceassociated parameters desired by the mathematical model. The following steps outline the approach used to attain estimates for the parameters $W_{s k}, L_{s k}, N_{s k}$ and $V_{s k}$ for each $s \in \mathcal{S}, k \in \mathcal{Q}$.

1. Information on movies to be screened and their showtimes is available for the planning horizon.

2. For each movie and showtime combination, demand distribution for the size of audience can be estimated using past data.

3. One can find the intermissions of those movies which will fall into each time-cell.

4. The concession stand operates as a queuing system for a given time-cell. If such a queuing system can be modeled and analyzed, the parameters considered in the mathematical model can be computed.

5. Note that the computation in step 4 should be repeated for each time-cell and for each possible number of workers considered. Specifically, one can estimate $W_{s k}, L_{s k}$, $N_{s k}$ and $V_{s k}$ for each $s \in \mathcal{S}, k \in \mathcal{Q}$.

Most of the staffing problems in the literature assume a continuous arrival rate for service, enabling the use of a steady-state analysis. In the venue management problem considered, on the other hand, there is a limited time (intermission) during which customers can be served. Therefore, steady-state analysis is not meaningful; a complete transient analysis is needed. Moreover, some customers are lost (customers may abandon) as the anxiety of waiting may increase closer to the end of intermission time. Additionally, customers come out almost simultaneously as a group at the intermission, and hence the queue has practically bulk arrivals. This is a hard queuing system to model and analyze.

A queuing model of the concession stand can be built under different assumptions. Once a reasonable model is available, having transient analysis compared to steady-state analysis will ease the computational requirements. One can model the problem using simulation, or an analytical model with some assumptions can be utilized. As a heuristic, we consider a simple analytical model to estimate the required parameters. Details of the model are given in the Appendix. Using the prescribed model in the Appendix, computations required in steps 4 and 5 of the outline can be carried out, and thus parameters of the mathematical model presented in Sect. 3 can be estimated.

\section{Computational complexity of the problem}

In this section, we provide the computational complexity status of the problem under consideration. Consider, without loss of generality, the simple case where workers of the same type with potentially different starting times are considered as different types. In other words, with a worker type, we identify a unique starting time at each working day, and specifically $\mathcal{Q}=\mathcal{T}$. For each $s \in \mathcal{S}$ and $t \in \mathcal{T}$, we let parameter

$A_{s}^{t}= \begin{cases}1 & \text { if worker } t \text { works in period } s \\ 0 & \text { otherwise }\end{cases}$

indicate the periods covered by each worker. Now it is possible to view our venue management problem as the following optimization problem:

$$
\begin{aligned}
\max & \sum_{s \in \mathcal{S}} \mathcal{V}_{s}\left(\sum_{t \in \mathcal{T}} A_{s}^{t} \lambda^{t}\right)-\sum_{t \in \mathcal{T}} C^{t} \lambda^{t} \\
\text { s.t. } & \sum_{t \in \mathcal{T}} A_{s}^{t} \lambda^{t} \geq \bar{\lambda}_{s} \quad s \in \mathcal{S} \\
& \lambda^{t} \geq 0, \quad \text { integer } \quad t \in \mathcal{T} .
\end{aligned}
$$

The decision version of the venue management problem, say DVMP, can be posed as (following the convention of Garey and Johnson 1979):

Instance $\mathcal{S}, \mathcal{T}, \mathcal{V}_{s}:\{1, \ldots, T\} \rightarrow \mathbb{R}_{\geq 0}$ and $\bar{\lambda}_{s}$ for each $s \in \mathcal{S}, C^{t}$ for each $t \in \mathcal{T}$ and $\mathcal{P}$.

Question Does there exist a worker schedule meeting the service level requirement with profit of at least $\mathcal{P}$ ?

Consider the following minimum cover problem (MC) which is known to be NP-complete (Garey and Johnson 1979).

Instance Collection $\mathcal{C}$ of subsets of a finite universe $\mathcal{U}$, positive integer $K \leq|C|$.

Question Does $\mathcal{C}$ contain a cover for $\mathcal{U}$ of size $K$ or less?

Theorem 2 DVMP is strongly NP-complete even if there are no service level requirements.

Proof Membership in NP is obvious, since checking the profit level of a worker schedule can be accomplished in polynomial time.

We proceed by showing that DVMP contains MC as a special case. Let $\mathcal{U}=\{1, \ldots, n\}, \mathcal{C}=\left\{O_{1}, \ldots, O_{m}\right\}$ 
and $K$ constitute an arbitrary instance of MC. Consider the following instance of DVMP. We identify periods with the universe and workers with the subsets. Specifically, let $\mathcal{S}=\{1, \ldots, n\}, \mathcal{T}=\{1, \ldots, m\}$,

$A_{s}^{t}= \begin{cases}1 & \text { if } s \in O_{t} \\ 0 & \text { otherwise }\end{cases}$

$C^{t}=1$ for each $t \in \mathcal{T}, \mathcal{P}=n-K$ and finally

$\mathcal{V}_{s}(q)= \begin{cases}1 & \text { if } \quad q \geq 1 \\ 0 & \text { otherwise. }\end{cases}$

Clearly, this instance of DVMP has a solution with profit of at least $n-K$ if and only if the MC instance has a cover of size at most $K$.

Since the special case of DVMP with no service level requirements, i.e., when $\bar{\lambda}_{s}=0 \forall s \in \mathcal{S}$, is NP-hard, so is DVMP. The following result considers the special case of DVMP when the revenue functions are linear.

Theorem 3 DVMP is strongly NP-complete even if the revenue functions $\mathcal{V}_{s}$ for $s \in \mathcal{S}$ are all linear.

Proof It is easy to see that DVMP belongs to NP, since given any worker schedule, checking whether the service level requirement is met at a certain profit level can be done in polynomial time.

We shall proceed as in the proof of Theorem 2 by showing that DVMP contains MC as a special case. Let $\mathcal{U}=$ $\{1, \ldots, n\}, \mathcal{C}=\left\{O_{1}, \ldots, O_{m}\right\}$ and $K$ constitute an arbitrary instance of MC. Consider the following instance of DVMP. We identify periods with the universe and workers with the subsets. In particular, let $\mathcal{S}=\{1, \ldots, n\}, \mathcal{T}=\{1, \ldots, m\}$,

$A_{s}^{t}= \begin{cases}1 & \text { if } s \in O_{t} \\ 0 & \text { otherwise }\end{cases}$

$\bar{\lambda}_{s}=1$ for each $s \in \mathcal{S}, C^{t}=\left|O_{t}\right|+1$ for each $t \in \mathcal{T}$, $\mathcal{P}=-K$ and finally $\mathcal{V}_{s}(q)=q$ for each $s \in \mathcal{S}$. With these specifications, model (18)-(20) becomes:

$$
\begin{array}{cl}
\max & -\sum_{t \in \mathcal{T}} \lambda^{t} \\
\text { s.t. } & \sum_{t \in \mathcal{T}: s \in O_{t}} \lambda^{t} \geq 1 \quad s \in \mathcal{S}, \\
& \lambda^{t} \geq 0, \quad \text { integer } \quad t \in \mathcal{T} .
\end{array}
$$

Clearly, this instance of DVMP has a solution with profit at least $-K$ if and only if MC instance has a cover of size at most $K$.
Note that if the revenue functions are linear, i.e., $\mathcal{V}_{s}(q)=$ $v_{s} q$ for $s \in \mathcal{S}$ where $v_{s} \in \mathbb{R}_{\geq 0}$, and there are no service level requirements, then model (18)-(20) reduces to:

$$
\begin{aligned}
\max & \sum_{t \in \mathcal{T}}\left(\sum_{s \in \mathcal{S}} v_{s} A_{s}^{t}-C^{t}\right) \lambda^{t} \\
\text { s.t. } & \lambda^{t} \geq 0, \quad \text { integer } t \in \mathcal{T} .
\end{aligned}
$$

The optimal solution will use each worker type indefinitely as long as the contribution to the objective is positive and hence can be identified easily in polynomial time.

\section{Computational results}

In this section, we provide the results of extensive computational experiments with the data for the case study in order to show the efficacy of the solution approach and to provide insights.

\subsection{Motivation and base data}

Our main motivation for performing some numerical analysis is to observe the advantages of using the relaxed version of the model. Even if we show in Sect. 5 that the problem is NP-complete, given certain real-life settings, we think that it might be reasonable to analyze the effect of several of the model attributes on the solution time. In this section, we present the results of the experiments we carried out.

The source of demand data that we use in the numerical experiments is a project experience conducted at a local movie theater center (Ahipasaoglu 2015). In our case study, the planning period is a week, each time-cell corresponds to an hour, and there are 14 time-cells in a day. Accordingly, hours of a day and days of the week are two main determinants of the demand data. We used a multiplicative model to represent the data: Define $H_{i j}$ as the relative weight of the demand for the $i$ th hour during the $j$ th day, and $D_{j}$ as the relative weight of the demand for the $j$ th day. Hence,

$\sum_{i} H_{i j}=$ number of hours worked in day $j, \quad j=1, \ldots, 7$

and

$\sum_{j} D_{j}=7$

The data used are given in Table 1. Note that the data show the properties mentioned in Sect. 4 and represent an average week. Of course, depending on the specific movies shown within the week, the pattern might change. 


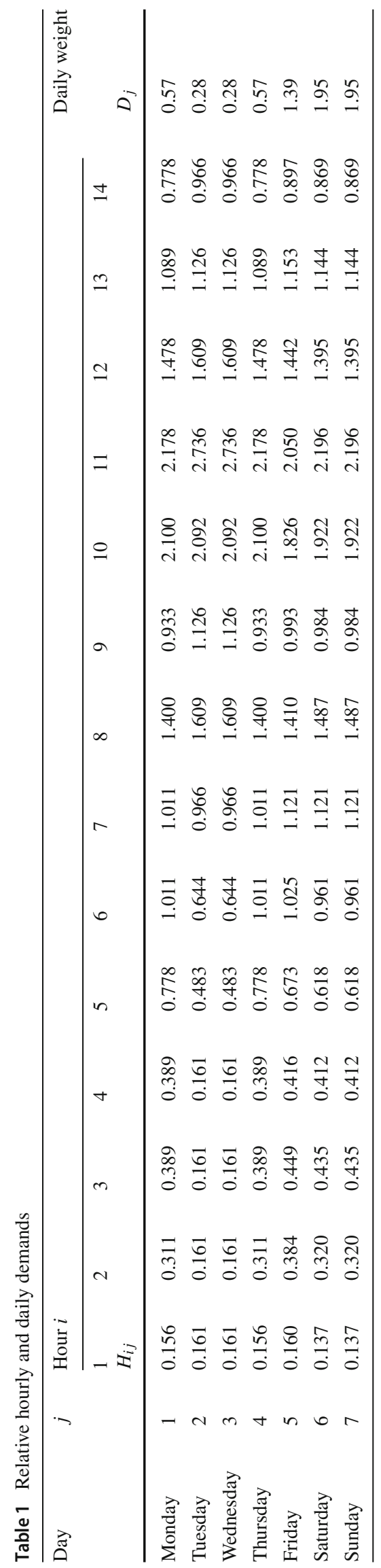

The remaining data were artificially generated according to the properties in Sect. 4. We used a concave function to represent the revenue. Let $\mathcal{V}_{i j}(k)$ denote the revenue function for the $i$ th hour during the $j$ th day, and let $k$ denote the number of workers working in this time-cell. The following function is used to represent the revenue:

$\mathcal{V}_{i j}(k)=a k+b k^{f} H_{i j} D_{j}$

where $a, b$ and $f$ are positive constants, with $f<1$.

\subsection{Experimental setup}

For the experiments, we need to specify a universal set for work patterns. We made three simplifications in order to restrict possibilities for work patterns:

- The hours worked in a day are always consecutive. Our model will determine the starting hours.

- Work patterns are designed so that in a given day, one can work $0,4,8$ or $10 \mathrm{~h}$.

- A worker should work at least $8 \mathrm{~h}$ in total during the week.

Possible work patterns are then distinguished according to

1. Number of working days per week

2. Number of working hours per working day

3. Actual days of working

4. Hours worked during the weekdays

5. Hours worked during the weekend

6. Hours of overtime (weekday or weekend).

As a result, we defined a total of 3148 work patterns. Note that this number does not include the start time for a worker in a given day; hence the actual number of choices is much greater.

We generated random instances that will select 100, 300 and 500 different work patterns among the 3148 available. A given random stream to generate 100 work patterns was used to generate the case with 300 (and 500) work patterns to ensure that the patterns included in smaller-sized problems were also included in the larger cases. This will ensure a smaller variance when comparing the results.

We assumed that workers are homogeneous, and hence for a standard hour they are paid exactly the same amount, call it c per hour. However, the actual hourly wage rate is calculated in the following manner:

1. Weekend rate per hour is twice the weekday rate per hour.

2. If one works more than $8 \mathrm{~h}$ a day, hours in excess of 8 are considered overtime hours.

3. Overtime rate per hour is 1.5 times the regular rate. 
As a result, one can compute the weekly wage of a worker with the given pattern.

For the experiments, we set $a=0.5, f=0.5, b$ as a uniformly distributed random variable in the range [8, 12], and $c$ as a uniformly distributed random variable in the range [ $\left.c_{\min }, c_{\max }\right]$, where $c_{\min }$ and $c_{\max }$ are selected to ensure that the generated problem does not have a trivial solutionemploy all the available workers in set $\mathcal{Q}$ at all times or result in extremely negative profits.

We solved the cases with different demand structures as well. Cases C1-C4 correspond to patterns D1-D4, respectively, where D1 is as given in Table 1-demand pattern with real data, $\mathrm{C} 2$ is the case where we increased the variability by increasing the proportion of the peak demand days/hours compared to D1. Case C3, on the other hand, is the case where variability compared to real data is lower, and finally, $\mathrm{C} 4$ is the case where demand values are taken to be identical for all time-cells.

We use the following procedure to generate our instances:

1. Select number of work patterns $(100,300,500)$

2. Generate 10 instances with 100 (or 300 or 500) work patterns

3. For each instance, generate 25 combinations of (b,c), five each

4. For each $(b, c)$ combination, use four different demand structures as specified above.

As a result, we obtain 3000 instances of the problem. Finally, for each instance, we first solved the problem without imposing any service level restrictions. Then, we added service level constraints by enforcing the condition that for each time-cell, a minimum number of workers (at least one more than the level obtained in the solution without the service level requirement ensuring that the introduced service level is always binding), bringing the total number of instances to 6000. Note that we used the same seed to generate instances, whenever appropriate, to control the variability induced by randomness. We solved our models using GAMS/Cplex 10.2 software on a workstation with an Intel 2.66-GHz Xeon processor and 8-GB memory.

\subsection{Effects of number of work patterns}

We analyze the results of random experiments using two measures: the first is the average CPU time (ACT), and the second is the variability in CPU times (VCT), which is defined as

$\mathrm{VCT}=\frac{(\max -\min )}{\mathrm{ACT}}$.

The values max and min are defined either with respect to the averages of 10 randomly generated instances as defined in step 2 of the generation procedure (we call these batch extremes), or using actual maximum and minimum values, as observed by those instances (we call these individual extremes). We use both definitions in analyzing the results. Note that a large VCT value implies not only that run-times will have a large variability, but also that run-times will have a tendency to be larger, as minimum time changes very little across the problem instances solved. Also note that VCT values would be larger if we did not control random effects in the instances generated.

\subsubsection{Comparison based on ACT}

We summarize the results for all instances in Table 2. With the "Integer Model," we refer to the IP specified by Eqs. (1)-(3), (8)-(10), (11) and (12), whereas with the "Relaxed Model" we have the same IP, while relaxing the binary requirements in (8). First, note that the average CPU time increases by $219 \%$ as the problem size increases from 100 to 300 work patterns, and $574 \%$ from 100 to 500 work patterns. A similar increase in average CPU time is observed for the relaxed problem. Note that the \% decrease in the average CPU times when we apply the relaxed problem is consistently lower, but differences are not large (last column of Table 2).

When we solve the instances with service constraint, on the other hand, the effect of the relaxed problem on CPU time can be better observed, as the problem with service level constraint is expected to be harder. We summarize the results for these instances in Table 3. First note that the growth in the average CPU time is $247 \%$ as the problem size goes from 100 work patterns to 300 work patterns, and $591 \%$ from 100 to 500. A similar growth in average CPU time is observed for the relaxed problem. Note that this result is very similar to what

Table 2 Average CPU times over 3000 instances

\begin{tabular}{llll}
\hline $\begin{array}{r}\text { \# of work } \\
\text { patterns }\end{array}$ & $\begin{array}{c}\text { Integer } \\
\text { model } \\
\text { ACT }(\mathrm{s})\end{array}$ & $\begin{array}{c}\text { Relaxed } \\
\text { model } \\
\text { ACT }(\mathrm{s})\end{array}$ & $\%$ Decrease \\
\hline 100 & 0.98 & 0.89 & 9.20 \\
300 & 3.12 & 2.94 & 5.77 \\
500 & 6.58 & 6.03 & 8.35 \\
\hline
\end{tabular}

Table 3 Average CPU times over 3000 instances with service constraints

\begin{tabular}{lccc}
\hline $\begin{array}{c}\text { \# of work } \\
\text { patterns }\end{array}$ & $\begin{array}{c}\text { Integer } \\
\text { model } \\
\text { ACT }(\mathrm{s})\end{array}$ & $\begin{array}{c}\text { Relaxed } \\
\text { model } \\
\text { ACT }(\mathrm{s})\end{array}$ & $\%$ Decrease \\
\hline 100 & 1.96 & 1.92 & 1.91 \\
300 & 6.80 & 5.52 & 18.88 \\
500 & 13.56 & 10.10 & 25.51 \\
\hline
\end{tabular}


Table 4 Percentage increase in ACT when service constraint is added

\begin{tabular}{lll}
\hline \# of work patterns & Integer model & Relaxed model \\
\hline 100 & 100.97 & 117.09 \\
300 & 118.34 & 87.97 \\
500 & 106.09 & 67.49 \\
\hline
\end{tabular}

we obtained for the problem without the service constraints. When we observe the \% decrease in the average CPU times when we apply the relaxed problem (last column of Table 3), we now observe that the decrease $\%$ increases sharply with problem size.

In Table 4, we provide a comparison of the results in Tables 2 and 3, to observe the effect of service level restrictions on CPU. Specifically in columns 2 and 3, we record the percentage increases in the average CPU times when we have service constraints. For the integer formulation, the increase is steady with respect to problem size, whereas for the relaxed problem, it decreases with problem size, a possible indication of performance enhancement brought about by the relaxed problem.

Finally, we check the effect of demand patterns on the CPU time. In all numerical results for $\mathrm{C} 2$, the case with greater variability than the real data, the average CPU times of the integer and relaxed problems were the lowest. This is expected, as the more variable the demand, the lower the number of competing work patterns to be utilized at optimality. Hence, we can solve our problems with smaller CPU times on average. We present these results in Table 5. As can be seen, the difference is about $12 \%$ for the integer problem (or $14 \%$ for the relaxed problem) for 100 work patterns. However, it is interesting to note that this difference decreases as we increase the number of work patterns, as can be followed from Table 5. Additionally, when we replicate the results of Table 5 for the case with service constraints (not tabulated), we observe that the differences are even much less. We think that this observation indicates that the effect of demand pattern is a lesser concern when we have larger-sized/harder problems.

\subsubsection{Comparison based on VCT}

We recorded maximum CPU times for any batch of runs. In all but a few cases, as expected, the maximums of the relaxed problem are much less than those of the integer problem. There are some exceptions, where maximum CPU time of a group of relaxed instances is relatively large. This is expected, as the problem is shown to be NP-hard in Sect. 5, and hence such instances should be observed. We believe, therefore, that the analysis of the numerical results using a variability measure is reasonable. We summarize the results for all instances in Table 6. Note that the $V C T$ measure defined by Eq. (21) considers whether the average achieved is in a reasonable range-in other words, can be considered as a measure of variability. In Table 6 , we present the results based on batch maximum values as well as individual maximums (both maximums averaged for service and non-service cases). The results are quite interesting and indicate the following:

- There is no obvious effect of problem size on VCT values. The second and third columns indicate that the values are very similar; the fifth and sixth columns may also indicate this, although there is more fluctuation. We believe that this observation makes the use of VCT values reasonable, as the variability measure considered can be said to be independent of the magnitude.

- We observe that the relaxed problem has smaller VCT values - the percentage decreases are presented in the table. This is an obvious benefit of the relaxed problem, indicating that the CPU times recorded have a greater tendency to be around the average than with the integer formulation.

- Finally, the VCT figures for individual extremes may suggest the difficulty of the problem, already indicated by the complexity results in Sect. 5. The values for certain instances may be arbitrarily large.

\subsection{Optimal revenue as a function of problem size}

In this section, we report the effect of problem characteristics on the objective function values. Recall that problem instances created for different numbers of work patterns are correlated, as we first generate an instance with 500 work patterns, and apply exactly the same selection of random parameters, as well as a subset of those 500 when we are solving an instance with a lower number of work patterns. Hence,

Table 5 Effect of demand pattern, C2 with no service constraint

\begin{tabular}{llllllr}
\hline $\begin{array}{l}\text { \# of work } \\
\text { patterns }\end{array}$ & $\begin{array}{c}\text { Integer model } \\
\text { ACT for all (s) }\end{array}$ & $\begin{array}{c}\text { Integer model } \\
\text { ACT for only } \\
\text { C2 (s) }\end{array}$ & \% Difference & $\begin{array}{c}\text { Relaxed model } \\
\text { ACT for all (s) }\end{array}$ & $\begin{array}{c}\text { Relaxed model } \\
\text { ACT for only } \\
\text { C2 (s) }\end{array}$ \\
\hline 100 & 0.98 & 0.86 & 12.24 & 0.89 & 0.76 & 14.61 \\
300 & 3.12 & 2.83 & 9.29 & 2.94 & 2.64 & 5.70 \\
500 & 6.58 & 6.16 & 6.38 & 6.03 & 5.47 \\
\hline
\end{tabular}


Table 6 Variability in CPU times over 6000 instances

\begin{tabular}{|c|c|c|c|c|c|c|}
\hline \multirow[t]{2}{*}{ \# of work patterns } & \multicolumn{3}{|c|}{ Batch extremes $^{\mathrm{a}}$} & \multicolumn{3}{|c|}{ Individual extremes ${ }^{\mathrm{a}}$} \\
\hline & $\begin{array}{c}\text { Integer } \\
\text { model } \\
\text { VCT }\end{array}$ & $\begin{array}{c}\text { Relaxed } \\
\text { model } \\
\text { VCT }\end{array}$ & $\%$ Decrease & $\begin{array}{c}\text { Integer } \\
\text { model } \\
\text { VCT }\end{array}$ & $\begin{array}{c}\text { Relaxed } \\
\text { model } \\
\text { VCT }\end{array}$ & $\%$ Decrease \\
\hline 100 & 1.96 & 1.43 & 27.06 & 10.88 & 9.59 & 11.89 \\
\hline 300 & 2.01 & 1.60 & 20.53 & 13.79 & 13.66 & 0.99 \\
\hline 500 & 1.94 & 1.13 & 41.79 & 13.86 & 4.83 & 65.13 \\
\hline
\end{tabular}

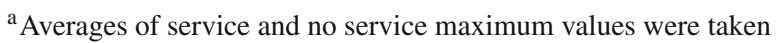

Table 7 Effect of including service levels

\begin{tabular}{ll}
\hline \# of work patterns & \% Decrease in the optimal value \\
\hline 100 & 26.18 \\
300 & 23.21 \\
500 & 22.82 \\
\hline
\end{tabular}

Table 8 Effect of number of work patterns on the objective function

\# of work patterns $\%$ Improvement in the objective function w.r.t. 100 work patterns

\begin{tabular}{lll}
\cline { 2 - 3 } & No service & With service \\
\hline 300 & 8.84 & 12.00 \\
500 & 16.68 & 21.17 \\
\hline
\end{tabular}

increasing the size of the problem by allowing more work patterns will guarantee a non-decreasing objective value. In Table 7, we report how service level constraint affected the objective value. Note that nearly stable percentage decreases indicate that we were able to generate instances which are comparable.

Finally, in Table 8 we show how the objective function improved when we allowed for more work patterns in the problem. Again, as expected, as we increase the number of patterns modeled in the problem, the objective function value improves. In the case where we have service constraint, the improvements are greater, indicating that there is more room for improvement.

Finally, when we analyze the effect of demand patterns on profitability (observed averages for 25 instances for each of the demand patterns, considering all runs with or without a service constraint), it was quite interesting to note the following: the profit obtained using the real-life demand pattern (case $\mathrm{C} 1$ ) was better than all the others in 18,17 , and 17 out of 20 cases for 100, 300 and 500 work patterns, respectively! Of course, there is no explanation for this fact, other than admiring the business side.

\section{Conclusions}

In this work, we have considered a shift planning approach for venue management. The case that motivates us (Ahipasaoglu 2015) is a concession stand in a movie theater, where demand is non-stationary and stochastic. Demand is known to be a random function of the showtime, day of the week and movie type, necessitating a bottom-up approach to estimate the total demand at any time interval. We propose a simple approach for estimating the expected concession revenue from movie customers during a single intermission given a workforce size, and build up the total revenue for all other movies and their intermissions if they coincide with the same time period. In our model, we identify worker types by the number of workdays per week and working hours per workday. This approach is flexible enough to incorporate different types of shift pattern requirements necessitated by regulations and ergonomic choices. Unlike the bulk of the existing literature, we schedule workforce so as to generate revenue. We provide an optimality cut for our model and, with the realistic assumption that the revenue generated will be a concave function of the workforce size, show that the binary requirement of a large number of variables can be relaxed in the search for the optimal solution. We also prove that the problem we are considering is NP-complete. We provide extensive computational results to show the effects of our proposed relaxation on computation time. We conclude that the proposed relaxation works well even under moderate problem sizes. Our computational results also provide some insight into the profit obtained when we allow for more worker types. We consider the effects of demand pattern on computation time as well as profit.

As an extension, we can generalize the model by allowing a continuous concave expected revenue function (as well as continuous service functions). One can show that our formulation coincides with a linear approximation of this general structure. The generalized formulation can handle the case where workers are allowed to be non-homogeneous. 


\section{Appendix}

The following assumptions were made on the basis of a project experience conducted at a local movie theater center (Ahipasaoglu 2015).

1. Venue managers have sufficient information to estimate the probability distribution of the incoming customers to the venue and the proportion of those asking for service at a concession stand as a function of (i) type of the movie, (ii) time of the day, (iii) day of the week, and (iv) week of the year.

2. It has been observed that the revenue obtained per customer served is a constant dependent only on the type of movie.

3. Some customers waiting for service tend to abandon if they are not served within a specified time. We assume that we can estimate the times and abandonment probabilities for such incidents as a function of queue size at those times.

4. We assume that the service time for a worker is an exponential random variable; if there are $Q$ workers (identical), we assume that the service rate will be $Q \mu$, where $\mu$ is the service rate for a worker.

5. We assume that once the intermission is over, the concession stand will close. One way to relax this assumption is by adding an additional time after the intermission is over.

6. We assume that customers arrive in bulk at the beginning of an intermission. This is an assumption made after observing the data. Of course, we can relax this assumption and consider a case where there might be more than one incidence for bulk arrivals during an intermission, which will only complicate computations.

7. We assume that there is only one intermission at a time. This assumption can be relaxed and, similar to assumption 6, we will only face more complicated computations.

Under these assumptions, we propose a simple model for computing the expected revenue from a single intermission. Our variables are as follows:

$\mu$ exponential service rate of a single worker

$Q$ number of workers

$Q \mu$ exponential service rate when there are $Q$ workers (approximation)

$\mathrm{NR}_{O}$ number of customers that arrive in bulk to the concession stand at the very beginning of the intermission (initial queue size)

$r$ number of abandonment incidents during an intermission, where $r \in\{0,1, \ldots, R\}$ and $r=0$ means that there is no abandonment $\tau_{i}$ time after arrival during which, if a customer is not served, he/she will consider abandonment, depending on the queue size, $i=1, \ldots, R$

$M_{i}$ critical queue size at $\tau_{i}$ for individual decision, $i=$ $1, \ldots, R$

$\mathrm{NS}_{i}$ number of customers served between $i-1$ st and $i$ th abandonment incidents, $i=1, \ldots, R$ (assume the beginning of the intermission corresponds to the 0th abandonment incident)

$\mathrm{NR}_{i}$ remaining number of customers in the queue immediately after the $i$ th abandonment incident, $i=1, \ldots, R$

$p_{i}$ probability for the $i$ th abandonment incident which occurs only if $\mathrm{NR}_{i-1}-\mathrm{NS}_{i}>M_{i}, i=1, \ldots, R$. In other words, if the number of customers in the queue, $\mathrm{NR}_{i-1}-\mathrm{NS}_{i}$, is greater than the threshold value, $M_{i}$, we will observe abandonment with probability $p_{i}$

Revenue $\sum_{i=1}^{R} a \mathrm{NS}_{i}$, where $a$ is assumed to be a constant revenue received per customer served for the specific movie type considered.

Note that other than the $Q \mu$ term, our decision variables in the scheduling problem will be a function of the movie type, time of day, day of the week, and week of the year. The estimation process seems to be exhaustive; however, most venues (or collections of venues) have most of the needed data for estimation. We consider a special case where $R=2$. Hence, we use a simpler rule for abandonment.

1. At time $\tau_{1}$, if $\mathrm{NR}_{0}-\mathrm{NS}_{1}>M_{1}$, then $\mathrm{NR}_{1}=\left(\mathrm{NR}_{0}-\right.$ $\left.\mathrm{NS}_{1}\right)\left(1-p_{1}\right)$. Note that $\mathrm{NR}_{1}$ may not be an integer. Without loss of generality, we replace $\left(\mathrm{NR}_{0}-\mathrm{NS}_{1}\right)(1-$ $\left.p_{1}\right)$ with $\left\lfloor\left(\mathrm{NR}_{0}-\mathrm{NS}_{1}\right)\left(1-p_{1}\right)\right\rfloor$, where $\lfloor x\rfloor$ is the floor operation taking the largest integer smaller than or equal to $x$.

2. At $\tau_{1}+\tau_{2}$, where $\tau_{1}+\tau_{2}$ is taken as the duration of the intermission, if $\mathrm{NR}_{1}-\mathrm{NS}_{2}>0$, then $\mathrm{NR}_{2}=0$, i.e., all unserved customers will leave the queue.

For a given number of workers, the following steps can be carried out for computations:

(i) Let the probability distribution for the initial number of customers asking for concession service be defined as $\operatorname{Pr}\left\{\mathrm{NR}_{0}=i\right\}, i \in \mathcal{I}=\{1, \ldots, I\}$, where $\mathcal{I}$ is the set of the possible number of concession customers.

(ii) $\left\{\mathrm{NS}_{1}=j\right\}$ is the event which says that $j$ customers are served during $\tau_{1}$ time units. Hence,

$\operatorname{Pr}\left\{\mathrm{NS}_{1}=j\right\}=\frac{e^{-Q \mu \tau_{1}}\left(Q \mu \tau_{1}\right)^{j}}{j !} j=0,1, \ldots, \mathrm{NR}_{0}-1$ 
Note that

$\operatorname{Pr}\left\{\mathrm{NS}_{1}=\mathrm{NR}_{0}\right\}=1-\sum_{j=0}^{\mathrm{NR}_{0}-1} \frac{e^{-Q \mu \tau_{1}}\left(Q \mu \tau_{1}\right)^{j}}{j !}$

In other words, this is a truncated Poisson process.

(iii) We would like to find the distribution of $N R_{1}$, which is dependent on the distribution of $\mathrm{NS}_{1}$. Note that there are at most two ways to arrive at an event $\left\{\mathrm{NR}_{1}=\right.$ $x$; ; we can have no abandonment and simply have $x$ customers remaining, or we can arrive at $x$ remaining customers after abandonment. Note that we take care of non-integer values by a consistent truncation. Let

$\mathrm{NR}_{1}^{\prime}$ : represent events with no abandonment

$\mathrm{NR}_{1}^{\prime \prime}$ represent events with abandonment

(a) Case with no abandonment $N S_{1} \geq N R_{0}-M_{1}$ :

$$
\begin{aligned}
\left\{\mathrm{NR}_{1}^{\prime}=x\right\} \equiv & \left\{\mathrm{NS}_{1}=\mathrm{NR}_{0}-x\right\} \text { and } \\
\operatorname{Pr}\left\{\mathrm{NR}_{1}^{\prime}=x\right\}= & \operatorname{Pr}\left\{\mathrm{NS}_{1}=\mathrm{NR}_{0}-x\right\} \\
& \text { for } x=0,1, \ldots, M_{1}
\end{aligned}
$$

(b) Case with abandonment $\mathrm{NS}_{1}<\mathrm{NR}_{0}-M_{1}$ :

We are interested in defining event

$$
\left\{\mathrm{NR}_{1}^{\prime \prime}=x\right\} \text { for } x=0, \ldots,\left\lfloor\left(1-p_{1}\right) N R_{0}\right\rfloor \text {. }
$$

Note that $\mathrm{NR}_{1}=\left\lfloor\left(\mathrm{NR}_{0}-\mathrm{NS}_{1}\right)\left(1-p_{1}\right)\right\rfloor$ is a function of the number served, $\mathrm{NS}_{1}$. $\mathrm{NS}_{1}$ may take different values but still $\mathrm{NR}_{1}$ may not change depending on the floor operation.

Define $\mathrm{NS}_{x}$ as the set of $\mathrm{NS}_{1}$ values that yield $x$, where $x=0, \ldots,\left\lfloor\left(1-p_{1}\right) \mathrm{NR}_{0}\right\rfloor$. Note that some $\mathrm{NS}_{x}$ may be empty.

$$
\left\{\mathrm{NR}_{1}^{\prime \prime}=x\right\}=\cup_{i \in \mathrm{NS}_{x}}\left\{\mathrm{NS}_{1}=i\right\}
$$

Note that the above events are independent. Hence,

$$
\operatorname{Pr}\left\{\mathrm{NR}_{1}^{\prime \prime}=x\right\}=\sum_{i \in \mathrm{NS}_{x}} \operatorname{Pr}\left\{\mathrm{NS}_{1}=i\right\} .
$$

Given that two events are independent, one can write

$$
\left\{\mathrm{NR}_{1}=x\right\}=\left\{\mathrm{NR}_{1}^{\prime}=x\right\} \cup\left\{\mathrm{NR}_{1}^{\prime \prime}=x\right\} .
$$

Therefore,

$$
\begin{aligned}
\operatorname{Pr}\left\{\mathrm{NR}_{1}=x\right\}= & \operatorname{Pr}\left\{\mathrm{NR}_{1}^{\prime}=x\right\}+\operatorname{Pr}\left\{\mathrm{NR}_{1}^{\prime \prime}=x\right\} \\
& \text { for } x=0, \ldots, \mathrm{NR}_{0} .
\end{aligned}
$$

(iv) The same approach can be repeated for the remaining time period $\tau_{2}$, and $\operatorname{Pr}\left\{\mathrm{NS}_{2}=x\right\}$ and $\operatorname{Pr}\left\{\mathrm{NR}_{2}=x\right\}$ can be found.

(v) All performance measures can be computed once the probabilities are determined. Note that we repeat the procedure for all $\mathrm{NR}_{0}$ values $\left(\mathrm{NR}_{0}=i, i \in \mathcal{I}\right)$ and for all numbers of workers $Q$.

\section{References}

Ahipasaoglu, O. (2015). Setting staffing levels at concession stands in movie theaters. In: IE 490 Technical Report, Department of Industrial Engineering, Bilkent University.

Aksin, Z., Armony, M., \& Mehrotra, V. (2007). The modern call center: A multi-disciplinary perspective on operations management research. Production and Operations Management, 16(6), 665688.

Alfares, H. K. (2004). Survey, categorization, and comparison of recent tour scheduling literature. Annals of Operations Research, 127(1), $145-175$.

Aykin, T. (2000). A comparative evaluation of modeling approaches to the labor shift scheduling problem. European Journal of Operational Research, 125(2), 381-397.

Boyer, V., Gendron, B., \& Rousseau, L.-M. (2013). A branch-and-price algorithm for the multi-activity multi-task shift scheduling problem. Journal of Scheduling, 17(2), 185-197.

Brusco, M. J., \& Jacobs, L. W. (2000). Optimal models for meal-break and start-time flexibility in continuous tour scheduling. Management Science, 46(12), 1630-1641.

Brusco, M. J., Jacobs, L. W., Bongiorno, R. J., Lyons, D. V., \& Tang, B. (1995). Improving personnel scheduling at airline stations. Operations Research, 43(5), 741-751.

Buffa, E. S., Cosgrove, M. J., \& Luce, B. J. (1976). An integrated work shift scheduling system. Decision Sciences, 7(4), 620-630.

Cezik, T., Gunluk, O., \& Luss, H. (2001). An integer programming model for the weekly tour scheduling problem. Naval Research Logistics, 48(7), 607-624.

Chapados, N., Joliveau, M., L'Ecuyer, P., \& Rousseau, L.-M. (2014). Retail store scheduling for profit. European Journal of Operational Research, 239(3), 609-624.

Chen, B. P. K. (2000). Staffing levels at the Auckland police communication centre. In: Technical Report, Department of Engineering Science, University of Auckland.

Dahmen, S., \& Rekik, M. (2014). Solving multi-activity multi-day shift scheduling problems with a hybrid heuristic. Journal of Scheduling, 18(2), 207-223.

Defraeye, M., \& Van Nieuwenhuyse, I. (2016a). A branch-andbound algorithm for shift scheduling with stochastic nonstationary demand. Computers \& Operations Research, 65, 149-162.

Defraeye, M., \& Van Nieuwenhuyse, I. (2016b). Staffing and scheduling under nonstationary demand for service: A literature review. Omega, 58, 4-25.

Ernst, A. T., Jiang, H., Krishnamoorthy, M., Owens, B., \& Sier, D. (2004a). An annotated bibliography of personnel scheduling and rostering. Annals of Operations Research, 127(1), 21-144.

Ernst, A. T., Jiang, H., Krishnamoorthy, M., \& Sier, D. (2004b). Staff scheduling and rostering: A review of applications, methods and models. European Journal of Operational Research, 153(1), 3-27.

Felici, G., \& Gentile, C. (2004). A polyhedral approach for the staff rostering problem. Management Science, 50(3), 381-393. 
Garey, M. R., \& Johnson, D. S. (1979). Computers and intractability: A guide to the theory of $N P$-completeness. New York: W. H. Freeman \& Co. ISBN 0716710447.

Hampshire, R. C., Jennings, O. B., \& Massey, W. A. (2009). A timevarying call center design via lagrangian mechanics. Probability in the Engineering and Informational Sciences, 23, 231-259, 4.

Helber, S., \& Henken, K. (2008). Profit-oriented shift scheduling of inbound contact centers with skills-based routing, impatient customers, and retrials. OR Spectrum, 32(1), 109-134.

Jacobs, L. W., \& Brusco, M. J. (1996). Overlapping start-time bands in implicit tour scheduling. Management Science, 42(9), 1247-1259.

Kabak, O., Ulengin, F., Aktas, E., Onsel, S., \& Topcu, Y. I. (2008). Efficient shift scheduling in the retail sector through two-stage optimization. European Journal of Operational Research, 184(1), 76-90.

Lam, S., Vandenbosch, M., \& Pearce, M. (1998). Retail sales force scheduling based on store traffic forecasting. Journal of Retailing, 74(1), 61-88.

Littler, R. A., \& Whitaker, D. (1997). Estimating staffing requirements at an airport terminal. Journal of the Operational Research Society, $48(2), 124-131$.

Love, R. R., \& Hoey, J. M. (1990). Management science improves fastfood operations. Interfaces, 20(2), 21-29.

Mani, V., Kesavan, S., \& Swaminathan, J. M. (2015). Estimating the impact of understaffing on sales and profitability in retail stores. Production and Operations Management, 24(2), 201-218.

Mason, A. J., Ryan, D. M., \& Panton, D. M. (1998). Integrated simulation, heuristic and optimisation approaches to staff scheduling. Operations Research, 46(2), 161-175.
Mattia, S., Rossi, F., Servilio, M., \& Smriglio, S. (2014). Combinatorial optimization, volume of 8596 lecture notes in computer science. Berlin: Springer.

Nobert, Y., \& Roy, J. (1998). Freight handling personnel scheduling at air cargo terminals. Transportation Science, 32(3), 295-301.

Rekik, M., Cordeau, J.-F., \& Soumis, F. (2004). Using benders decomposition to implicitly model tour scheduling. Annals of Operations Research, 128(1), 111-133.

Ren, Z. J., \& Zhou, Y.-P. (2008). Call center outsourcing: Coordinating staffing level and service quality. Management Science, 54(2), 369-383.

Sarin, S. C., \& Aggarwal, S. (2001). Modeling and algorithmic development of a staff scheduling problem. European Journal of Operational Research, 128(3), 558-569.

Schindler, S., \& Semmel, T. (1993). Station staffing at Pan American World Airways. Interfaces, 23(3), 91-98.

Stern, H. I., \& Hersh, M. (1980). Scheduling aircraft cleaning crews. Transportation Science, 14(3), 277-291.

Thompson, G. M. (1995). Improved implicit optimal modeling of the labor shift scheduling problem. Management Science, 41(4), 595607.

Thompson, G. M. (1997). Labor staffing and scheduling models for controlling service levels. Naval Research Logistics, 44(8), 719740 .

Van den Bergh, J., Beliën, J., De Bruecker, P., Demeulemeester, E., \& De Boeck, L. (2013). Personnel scheduling: A literature review. European Journal of Operational Research, 226(3), 367-385.

van Veldhoven, S., Post, G., van der Veen, E., \& Curtois, T. (2016). An assessment of a days off decomposition approach to personnel shift scheduling. Annals of Operations Research, 239(1), 207-223. 